\title{
Theoretical Justification of Single-Ended Dislocation-Source-Controlled Deformation of Micropillar fec Crystals
}

\author{
Shin Takeuchi, ${ }^{1}$ Keiichi Edagawa, ${ }^{2}$ and Yasushi Kamimura $\odot^{2, *}$ \\ ${ }^{1}$ Professor Emeritus of Tokyo University of Science, Shinjuku-ku, Tokyo 162-8601, Japan \\ ${ }^{2}$ Institute of Industrial Science, The University of Tokyo, Meguro-ku, Tokyo 153-8505, Japan
}

(Received 14 January 2021; revised 13 March 2021; accepted 19 March 2021; published 13 April 2021)

\begin{abstract}
It was established at the beginning of the 21st century that the critical resolved shear stress of small-sized (diameter from $50 \mathrm{~nm}$ to $10 \mu \mathrm{m}$ ) metallic crystals fabricated from bulk crystals increases drastically with decreasing specimen diameter. Dou and Derby [Scr. Mater. 61, 524 (2009)] showed that, the critical shear stresses of small-sized single crystals of various fcc metals obeyed a universal power law of specimen size with an exponent of -0.66 . In this study, we succeeded in reproducing almost perfectly the above universal relation without any adjustable parameters, based on a deformation process controlled by the operation of single-ended dislocation sources.
\end{abstract}

DOI: 10.1103/PhysRevLett.126.155501

It has long been known that thin crystals of whiskers exhibit anomalously high strengths with decreases in diameter, e.g., Ref. [1]. This phenomenon was first attributed to the lack of dislocations, but later interpreted in terms of the decrease in the dislocation source length with the decrease in specimen size. From the end of the 20th century, the focused ion beam (FIB) milling technique has been utilized to fabricate small deformation specimens with diameters ranging from a few micrometers down to the order of $100 \mathrm{~nm}[2,3]$. Thus, many systematic experiments on the size dependence of the critical resolved shear stresses (CRSSs) of small-sized specimens were performed until the end of the first decade of the 21st century; see the early review by Greer [4], a comprehensive review by Greer and De Hosson [5], and a recent review by Shahbeyk et al. [6].

In this Letter, we focus on pure fcc metals, where the friction stress to dislocation glide is negligible due to the low Peierls stress of the dissociated dislocation and the production of double-ended dislocation source by the double-cross slip mechanism is difficult due to the dislocation dissociation. The main features of deformation of micropillar fcc crystals are (i) stress-strain curves are discontinuous, consisting of flat portions separated by small steps $[2,5,7,8]$, as in other small-sized metallic crystals. This fact indicates that the yield stress is controlled by the dislocation source activation and the plastic strain is brought almost solely by the operation of a single source

Published by the American Physical Society under the terms of the Creative Commons Attribution 4.0 International license. Further distribution of this work must maintain attribution to the author(s) and the published article's title, journal citation, and DOI. with little work hardening by the activity of matrix dislocations; (ii) the CRSSs are largely scattered, but they increase drastically with decreases in specimen diameter $D$. By collecting the experimental data on single crystals of fcc metals including $\mathrm{Al}, \mathrm{Au}, \mathrm{Cu}$, and $\mathrm{Ni}$, Dou and Darby [9] showed that the CRSS normalized by the shear modulus $G$ can be expressed by a universal power-law function of the diameter $D$ normalized by the magnitude of the Burgers vector $b$, as given by Eq. (1) with the exponent $m \approx-0.66$ and the prefactor $A \approx 0.71$.

$$
\tau_{c} / G=A(D / b)^{m} .
$$

However, due to the large scatter of the data, assignment of the exponent cannot be done strictly; Uchic et al. obtained $m \approx 0.6$ for the same fcc crystals [10].

A lot of systematic experiments on size effects in metallic crystals have been performed not only on fcc metals but also on bcc metals, etc. [5]. It should be noted that in the classical experiments on metallic nanowires such wires fractured near the theoretical shear strength of the material, which is much higher than the yield stresses of nanopillars, regardless of the diameter because of the lack of dislocations [11]. In contrast to grown whiskers, fabricated nanopillar specimens contain rather high-density dislocations of $10^{12}-10^{14} \mathrm{~m}^{-2}$, forming braids or bundles. The established relation between the dislocation density $\rho$ and the flow stress, known as the Taylor relation, is given by

$$
\tau_{\rho}=\alpha G b \sqrt{\rho},
$$

with the coefficient $\alpha$ of $0.2-0.5$. Using $\alpha=0.3$ for lightly deformed crystals, $\tau_{\rho}$ is calculated as $10^{-4}-10^{-3} \mathrm{G}$ for $\rho=10^{12}-10^{14} \mathrm{~m}^{-2}$. The following fact should also be 


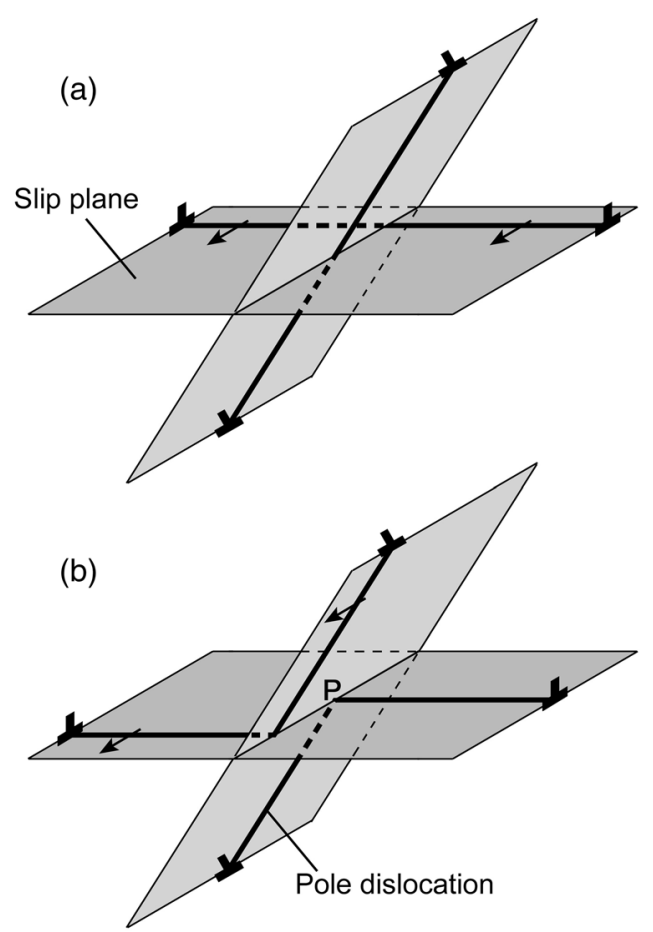

FIG. 1. A possible production process of a single-ended source. An edge dislocation on a primary slip plane is cut by an edge dislocation with the same Burgers vector on a cross slip plane to form a single-ended source. (a) Before cutting and (b) after cutting.

mentioned. Jennings et al. [12] showed that $\mathrm{Cu}$ nanopillars fabricated without FIB milling procedure containing dislocations of the order of $10^{14} \mathrm{~m}^{-2}$ show the same size dependence as those produced by the FIB milling process, thereby excluding the possibility of any special role of ion-beam produced defects. Thus, rather high density of dislocations is a key factor for the deformation of nanopillars.

The mechanism of strengthening with decreasing specimen size has been investigated, and various mechanisms have been proposed. Greer and Nix proposed the dislocation starvation effect [8], where with a decrease of the specimen size, dislocations rapidly exit the crystal surface, leading to the dislocation-starved state; the dislocation multiplication source necessary to continue the deformation is difficult to be produced in small specimens. On the other hand, based on the transmission electron microscope observations showing that the average dislocation density increases with decreasing crystal size, Norflect et al. [13] pointed out that forest hardening contributes to the strengthening of small specimens. Parthasarathy et al. performed a statistical analysis of the deformation process of small-sized crystals controlled by the multiplication process from truncated single-ended dislocation sources and the results are compared with experimental results [14]. Rao et al. [15] performed a large-scale 3D discrete dislocation simulation (DDD) for fcc Ni microcrystals of sizes $0.5-20 \mu \mathrm{m}$, introducing the three Frank-Read source densities of $7 \times 10^{11}, 2 \times 10^{12}$, and $10^{13} \mathrm{~m}^{-2}$. The results obtained showed characteristic stressstrain curves and power-law relations between the cell diameter and the critical resoled shear stress (CRSS), almost reproducing those observed in experiments. They argued that, among various hardening mechanisms, including source-truncation hardening, exhaustion hardening, forest hardening, nucleation-controlled size effect, etc., the sourcetruncation mechanism and exhaustion hardening are sufficient to explain the experiments. Following these studies, further detailed experiments, theories and 3D-DDD simulations have been conducted on the size effect of crystal strength until today [16-22], but the physical mechanism of it has not been fully clarified.

Now, we would like to point out the characteristic difference between the double-ended source controlled deformation and the single-ended source controlled deformation. In the former case, the critical shear stress is given by

$$
\tau_{c}^{d}=\beta G b / L+\alpha G b \sqrt{\rho},
$$

where $L$ is the source length and the numerical factor $\beta \approx 1$. The first term in the right side corresponds to the necessary bow-out stress and the second term is the resistance by the matrix dislocations during the bow-out process. In the latter case

$$
\tau_{c}^{s}=\left(\beta^{\prime} G b / L\right) \ln \left(L / r_{0}\right)+\alpha G b \sqrt{\rho},
$$

where $r_{0}$ is the cutoff radius of the dislocation core and will be replaced by $b$ hereafter. The difference from the former case is the presence of the factor $\ln \left(L / r_{0}\right)$, which comes in due to the free surface effect on the self-energy of bow-out dislocation [23]. If we neglect the dislocation hardening term, the source length dependence in the former case is $\tau_{c}^{d} \propto L^{-1}$, but in the latter case, the presence of the factor $\ln \left(L / r_{0}\right)$ in the first term in the right must decrease the absolute value of exponent of $L$, and hence assuming $L \propto D$, Eq. (1) may be justified. This is the motivation of the present theoretical calculations. In the source truncation theory by Parthasarathy et al., they used Eq. (3) instead of Eq. (4) and hence the theory is not based on the singleended source model. As a result, the obtained exponent $m$ [Eq. (1)] was close to -1 in the small $D$ region [see theoretical lines drawn in Figs. 3(a) and 3(b) in Ref. [14] ].

Single-ended dislocation sources that control the deformation may already be present in specimens or produced during preyield microstrain stage. Equation (1) holds for micropillar specimens with diameters down to $0.1 \mu \mathrm{m}$ and a single slip in a single crystal occurs by the activation of one of the twelve $\langle 110\rangle\{111\}$ slip systems, even in the smallest sample. Hence, in the former case, at least twelve single-ended sources should exist in the specimen. If we assume that the number of the preexisting sources in the 


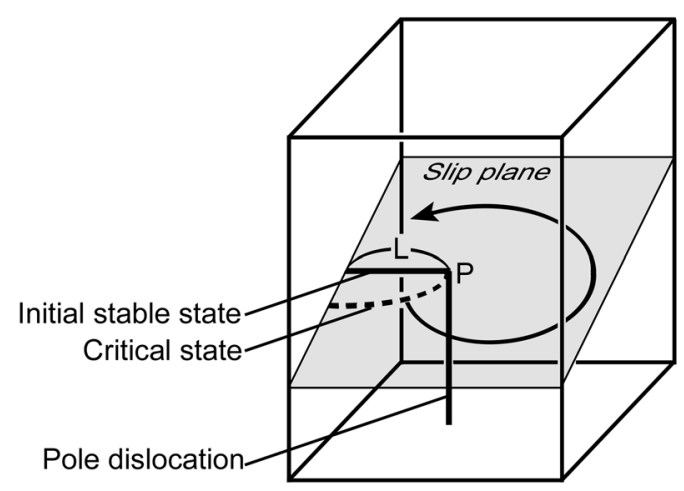

FIG. 2. Schematic of the stable state of surface-truncated single-ended dislocation source in square specimen.

specimen is proportional to the specimen volume, there should be quite a large number of sources in specimens with larger diameters; then, yielding should occur by the operation of the weakest source among the possible sources of the primary slip system. On the other hand, in the latter case, a possible production process of a single-ended source in the preyield microstrain stage is the cutting of an edge dislocation on the primary slip plane by an edge dislocation with the same Burgers vector on a cross slip plane, as depicted in Fig 1. Note that nanopillar specimens generally contain rather high-density dislocations so that such a process could occur even in very small specimens. Then, the pole position of the single-ended source to be activated may be located at random position on the slip plane. Hence, the expected CRSS should be widely scattered around the average value of the CRSS of randomly positioned single-ended dislocation poles on the slip plane.

In our calculations of CRSSs by the activation of singleended dislocation sources, the basic assumptions are (i) in pure fcc metals, the dislocation glide is practically athermal, (ii) the CRSS is determined by the activation of a single-ended source positioned at random on a slip plane, which has reached a stable configuration shown in Fig. 2. Under these conditions, we calculate the expected value of the critical yield stress by averaging the strengths of singleended sources positioned randomly over the slip plane.

Neglecting the matrix hardening term, the critical stress to activate a single-ended source positioned $L$ from the nearest side surface has been calculated $[23,24]$ and is given by

$$
\tau_{c}(L)=\left(\beta^{\prime} G b / L\right) \ln (L / b),
$$

where the coefficient $\beta^{\prime}$ depends on the dislocation character of the initial stable state of the single-ended dislocation. The coefficient $\beta^{\prime}$ was calculated to be in the range of $\beta^{\prime}=0.108 \pm 0.04$ by Pichaud et al. [23] by the iterative calculation of the segmented dislocation model for the bow-out process. The results of the 3D discrete dislocation dynamics simulation for the strength of single-ended dislocation source by Rao et al. [24] agree with those of Pichaud et al. within $15 \%$. In the following calculation, we use $\beta^{\prime}=0.108$ as the average value of a randomly oriented source dislocation. Equation (3) was obtained for the flat side surface, but we also apply the same equation for curved side surface of round specimen as an approximation.

At first, we calculate for the square pillar specimen of width $D$. Figure 3(a) represents the first quadrant of the slip plane at an angle of $45^{\circ}$ to the stress axis. The equally spaced bent lines in the figure indicate contour lines of equal strength of the single-ended source. The position of the pole is assumed to be located at any position with equal probability, excluding a thin surface layer of thickness $\delta$,
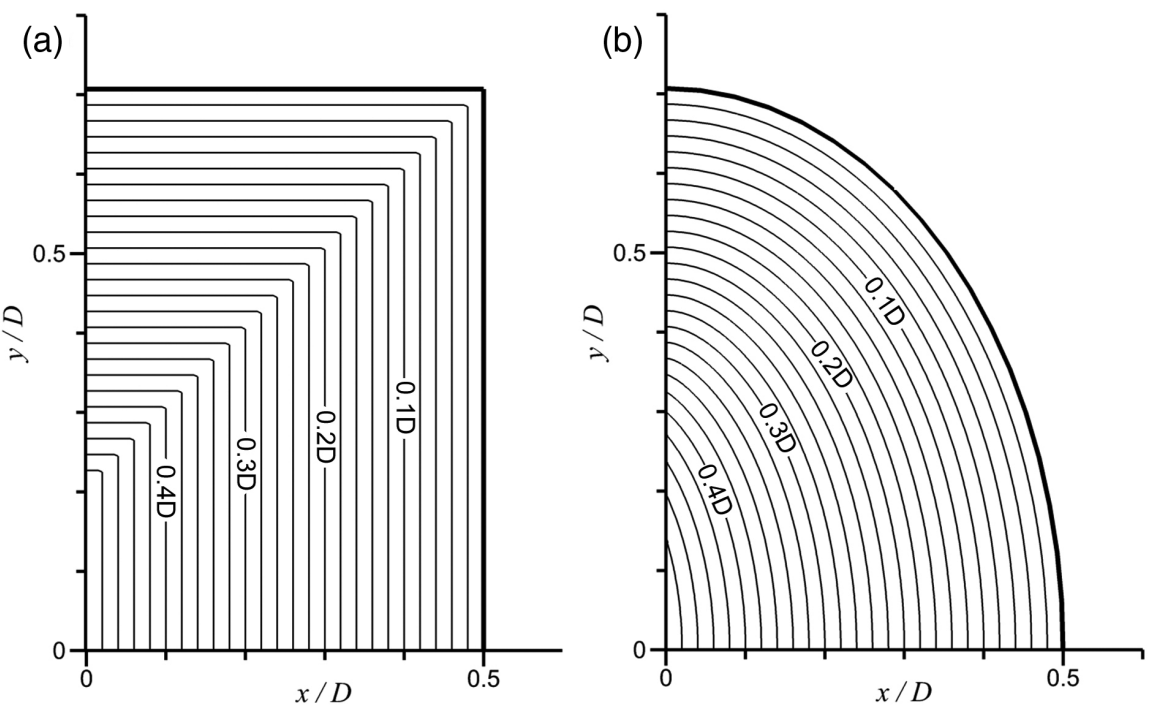

FIG. 3. (a) The first quadrant of the slip plane in a square pillar specimen, and (b) that in a round pillar specimen. In both figures, contour lines of equal strengths of the single-ended sources are drawn and are labeled by the $L$ value in units of $D$. 
where the pole dislocation cannot be stably positioned without escaping from the surface. In the following calculations we assume $\delta=5 b$. We note that if we use $\delta=20 b$, the overall results are essentially unchanged [25]. In this situation, the expected activation stress of the singleended source can be obtained by the following integration equation by use of Eq. (5):

$$
\frac{\tau_{c}}{G}=\frac{\int_{\delta}^{D / 2}\left\{\frac{0.108 b}{L} \ln \left(\frac{L}{b}\right) T(L)\right\} d L}{\int_{\delta}^{D / 2} T(L) d L},
$$

where $T(L)=2(\sqrt{2}+1) D-8 L$ is the length of the contour line at $L$ from the surface [Fig. 3(a)]; the poles positioned on this line possess the same strength. The denominator in the right-hand side of Eq. (6) corresponds to the area of the rectangular slip plane excluding the surface region with width $\delta$, i.e., $(\sqrt{2} D-2 \delta)(D-2 \delta)$. The integral in the numerator in the right-hand side of Eq. (6) can be calculated analytically, and $\tau_{c} / G$ vs $D / b$ relation calculated for $D$ value from $10^{2} b$ to $10^{5} b$ is shown by the red line in the log-log scale in Fig. 4.

Many of the experiments on sub-micrometer-sized specimens have been performed using round pillar specimens. In these cases, the slip planes are elliptical, and the expected values of the activation stresses of single-ended sources cannot be calculated analytically. Therefore, we divided the slip plane surface into meshes of the size $D / 2000$ and calculated in computer numerically by

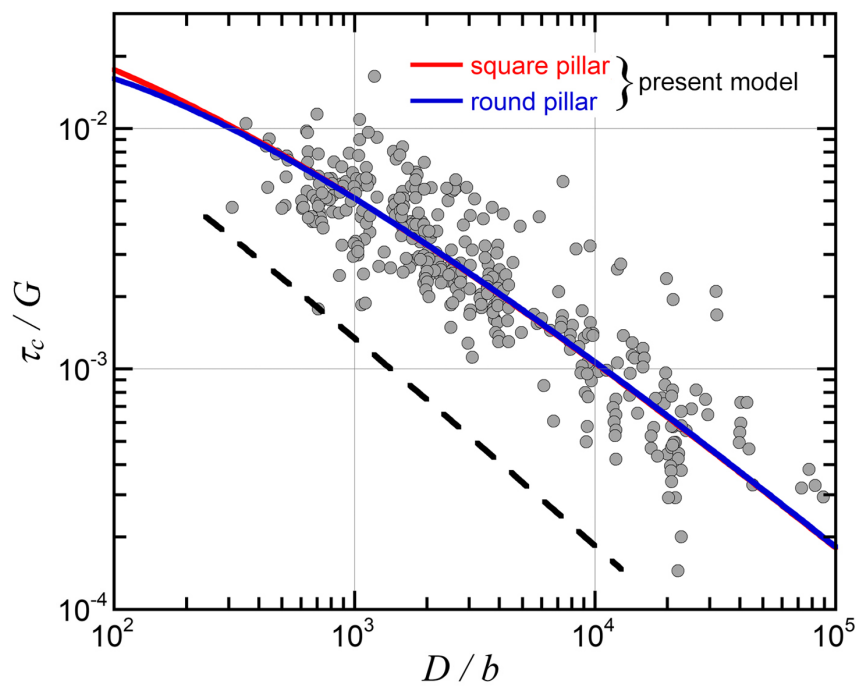

FIG. 4. Log-log plot of CRSS $\tau_{c}$ normalized by shear modulus $G$ as a function of the pillar size $D$ normalized by Burgers vector $b$ : Red line and blue lines show the calculated average values for square pillar specimen and round pillar specimens, respectively. Experimental results for fcc metals $\mathrm{Al}, \mathrm{Au}, \mathrm{Cu}$, and $\mathrm{Ni}$, collected by Shahbeyk et al. [6], are plotted by gray marks. The size dependence of the minimum value of CRSS is drawn by a dashed line. calculating the nearest distance from every mesh point inside the ellipsoid to the surface, excluding those in the surface layer of width $\delta$, calculating the strength of the single-ended source at each mesh point, and finally averaging the strengths to obtain the expected strength of a single-ended source in round pillar specimens of various cross-sectional diameters $D$. The calculated $\tau_{c} / G$ vs $D / b$ relation of the round pillar specimens is very close to that of square pillar specimens; the plotted line in blue overlaps with that for square pillars drawn in red in Fig. 4. The coincidence of the two lines arises from the fact that the ratio of the length of the contour lines of equal source strength in two slip planes is almost the same as the ratio of the slip-plane surface areas [25].

In Fig. 4, the experimental results for the fcc metals $\mathrm{Al}$, $\mathrm{Au}, \mathrm{Cu}$, and $\mathrm{Ni}$ collected by Shahbeyk et al. [6] are plotted by gray marks, which are reproduced very well by the calculated curves. The average slope of the curves between $D / b=2 \times 10^{2}$ and $D / b=2 \times 10^{4}$ is -0.66 , which happens to be exactly the same as that obtained by Dou and Darby [9]. From this agreement between the calculated results and experimental data, we can conclude that the randomly positioned single-ended sources control the size dependence of the CRSS of small-sized fcc crystals. In Fig. $4, \tau_{c} / G$ calculated for the weakest source is shown by a dashed line, which is located far below the experimental data. This clearly indicates that the deformation mechanism controlled by the weakest preexisting source is not suitable.

The normalized frequency distributions of the source strengths calculated for $D / b=10^{3}$ and $10^{4}$ are presented in Fig. 5. Both exhibit considerably large ranges of $\tau_{c} / G$ distributions exceeding an order of magnitude; this is qualitatively consistent with the large scatter observed in the experimental data in Fig. 4. However, the strength distribution for each size is highly asymmetrical in $\log \left(\tau_{c} / G\right)$, where the peak value is smaller than the average value shown in Fig. 4. As a result, the peak values of $\tau_{c} / G$ in Fig. 5 appear to be slightly lower than those of the

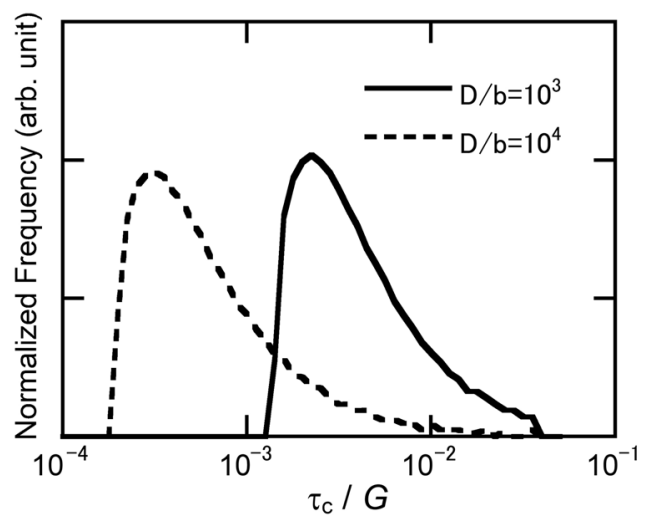

FIG. 5. Normalized distributions of calculated strengths of single-ended source for specimens of $D / b=10^{3}$ and $10^{4}$. 
experimental data in Fig. 4. We note here that the calculated values do not include the dislocation hardening component $\tau_{\rho}$, which should affect the bow-out process of the source dislocation; $\tau_{\rho} \approx 10^{-4}-10^{-3} G$ should be added to the calculated $\tau_{c}$.

In conclusion, we have presented a theoretical model based on single-ended dislocation-source controlled deformation to account for the specimen size dependence of the CRSSs so-far obtained experimentally for micropillar fcc crystals. In the model, we assumed that single-ended dislocation sources are produced during the preyield microstrain stage. Then, the expected CRSS was given by the average value of the source strengths of randomly positioned dislocation poles on the slip plane. The calculated average CRSS reproduced remarkably well the universal power-law behavior of the experimental data without any adjustable parameters.

*Corresponding author. yasushi@iis.u-tokyo.ac.jp

[1] S. S. Brenner, J. Appl. Phys. 27, 1484 (1956).

[2] M. D. Uchic, D. M. Dimiduk, J. N. Florando, and W. D. Nix, Science 305, 986 (2004).

[3] M. D. Uchic and D. M. Dimiduk, Mat. Sci. Eng. A 400-401, 268 (2005).

[4] J. R. Greer, Rev. Adv. Mater. Sci. 13, 59 (2006).

[5] J. R. Greer and J. T. M. De Hosson, Prog. Mater. Sci. 56, 654 (2011).

[6] S. Shahbeyk, G. Z. Voyiadjis, V. Habibi, S. H. Astaneh, and M. Yaghoobi, Crystals 9, 591 (2019).

[7] D. M. Dimiduk, M. D. Uchic, and T. A. Parthasarathy, Acta Mater. 53, 4065 (2005).
[8] J. R. Greer and W. D. Nix, Phys. Rev. B 73, 245410 (2006).

[9] R. Dou and B. Derby, Scr. Mater. 61, 524 (2009).

[10] M. D. Uchic, P. A. Shade, and D. M. Dimiduk, JOM 61, 36 (2009).

[11] S. S. Brenner, Science 128, 569 (1958).

[12] A. T. Jennings, M. J. Burek, and J. R. Greer, Phys. Rev. Lett. 104, 135503 (2010).

[13] D. M. Norfleet, D. M. Dimiduk, S. J. Polasik, M. D. Uchic, and M. J. Mills, Acta Mater. 56, 2988 (2008).

[14] T. A. Parthasarathy, S. I. Rao, D. M. Dimiduk, M. D. Uchic, and D. R. Trinkle, Scr. Mater. 56, 313 (2007).

[15] S. I. Rao, D. M. Dimiduk, T. A. Parthasarathy, M. D. Uchic, M. Tang, and C. Woodward, Acta Mater. 56, 3245 (2008).

[16] A. Rinaldi, Nanoscale 3, 4817 (2011).

[17] R. Gu and A. H. W. Ngan, Acta Mater. 60, 6102 (2012).

[18] R. Gu and A. H. W. Ngan, J. Mech. Phys. Solids 61, 1531 (2013).

[19] J. A. El-Awady, Nat. Commun. 6, 5926 (2015).

[20] H. Song, D. Dimiduk, and S. Papanikolaou, Phys. Rev. Lett. 122, 178001 (2019).

[21] J. Shin, L. Y. Chen, U. T. Sanli, G. Richter, S. Labat, M.-I. Richard, T. Cornelius, O. Thomas, and D. S. Gianda, Acta Mater. 166, 572 (2019).

[22] W. Liu, Y. Liu, Y. Cheng, L. Chen, L. Yu, X. Yi, O. Thomas, and H. Duan, Phys. Rev. Lett. 124, 235501 (2020).

[23] B. Pichaud, F. Minari, and J. Kellerhals, Philos. Mag. A 38, 593 (1978).

[24] S. I. Rao, D. M. Dimiduk, M. Tang, M. D. Uchic, T. A. Parthasarathy, and C. Woodward, Philos. Mag. 87, 4777 (2007).

[25] See Supplemental Material at http://link.aps.org/supplemental/ 10.1103/PhysRevLett.126.155501 for further calculation results concerning the effect of the surface layer thickness and the comparison between square and round specimens. 